\title{
OSG-Frakturen Weber-B/C: was ist Standard?
}

\author{
Alice Wichelhaus, Thomas Mittlmeier
}

\section{Zusammenfassung}

Frakturen des oberen Sprunggelenks (OSG) sind sehr häufige Verletzungen. Entscheidend für das klinische Ergebnis ist die richtige Klassifikation und Therapie. In der Regel werden Sprunggelenkfrakturen nach der Danis-Weber- und der Lauge-Hansen-Klassifikation eingeteilt. Entscheidend ist es, neben der Fraktur die verletzten Bandstrukturen zu erkennen und zu behandeln. Ziel ist eine möglichst anatomische Rekonstruktion der Gelenkflächen und der Stellung der Sprunggelenkgabel sowie eine Wiederherstellung der Stabilität der Syndesmose, um eine frühzeitige Arthroseentwicklung zu vermeiden.

\section{Standards in therapy of ankle fractures type Weber B/C}

Ankle fractures are a very common injury. The clinical outcome essentially depends on correct fracture classification and knowledge of the therapy regime. Ankle fractures are classified according to Danis-Weber or LaugeHansen. An important prognostic step is to identify all injured ankle components including the ligaments. Goal of any surgical treatment is an anatomic reduction of articular surfaces and the ankle mortise with special focus on syndesmotic stability in order to prevent osteoarthritis.

\section{Einleitung}

Frakturen des oberen Sprunggelenks (OSG) gehören zu den häufigsten Frakturen des Menschen. In Deutschland wurden im Jahr 2012 fast 75000 Patienten stationär unter dieser Diagnose behandelt. Personen jeden Alters sind betroffen, das Durchschnittsalter liegt bei knapp über 50 Jahren. Meist entsteht eine Sprunggelenkfraktur durch ein indirektes Trauma durch Umknicken auf unebenem Untergrund, häufig im Rahmen von Sportverletzungen oder Freizeitaktivitäten wie Laufen oder Springen. In einem Drittel der Fälle sind Alkoholeinfluss und rutschige Flächen als Mitverursacher feststellbar. Wichtig ist es, das Verletzungsausmaß nicht zu unterschätzen. Hierfür müssen alle am oberen Sprunggelenk beteiligten anatomischen Strukturen beurteilt werden. Dazu gehört außer dem Außen- und In-

OP-JOURNAL 2014; 30: 66-75

(c) Georg Thieme Verlag KG Stuttgart · New York DOI http://dx.doi.org/10.1055/s-0034-1383218

nenknöchel das Sprungbein, der äußere Seitenbandapparat, die dorsolaterale Tibiakante (Volkmann-Dreieck), die ventrolaterale Tibiakante (Tubercule de Chaput), das innere Seitenband, die Gelenkkapsel, die proximale Fibula (Verletzungstyp Maisonneuve) und die Syndesmosenbänder sowie die Membrana interossea. Der begleitende Weichteilschaden hat ebenfalls eine Auswirkung auf das Behandlungsergebnis.

\section{Fraktureinteilung}

\section{Klassifikation nach Danis-Weber (1966)}

Die im klinischen Alltag gebräuchlichste Klassifikation ist die nach Weber, die auch die Grundlage der AO-Klassifikation bildet. Hierbei wird die Frakturklassifikation nach anatomisch-pathologischen Kriterien anhand des Röntgenbilds vorgenommen. Sie bezieht sich ausschließlich auf die Höhe der Fibulafraktur in Bezug zur Syndesmose, ist einfach anwendbar, aber berücksichtigt nicht die Verletzung der medialen Strukturen. Damit ist keine sichere Aussage zur Stabilität möglich, mit Ausnahme der Weber-C-Frakturen, die grundsätzlich als instabil gelten. Eine sichere Aussage zur OP-Indikation lässt sich anhand dieser Klassifikation, insbesondere bei den häufigen Typ-B-Frakturen, nicht treffen.

\section{Weber-B-Fraktur (Abb. 1)}

- schräge und spiralförmige Fibulafrakturen mit Beginn auf Höhe der vorderen Syndesmose

- weiter Bereich von Verletzungsursachen ohne Differenzierung der verschiedenen Mechanismen und Aussage über die jeweils erforderliche Therapieform

- Weber-B entspricht nach Lauge-Hansen

- Pronations-Abduktions- (ab Schweregrad 3) oder

- Supinations-Eversions-Verletzungsmuster (ab Schweregrad 2)

\section{Weber-C-Fraktur (Abb. 2)}

- alle Fibulafrakturen oberhalb der Syndesmose

- Weber C entspricht Pronations-Eversions-Verletzungsmuster (ab Schweregrad 3) nach Lauge-Hansen

- Therapie: grundsätzlich operativ

Bei kombinierter Innenknöchelfraktur wird von einer bimalleolaren Fraktur gesprochen, bei gleichzeitig vorliegender Fraktur der dorsalen Tibia von einer trimalleolaren Fraktur. Die Fraktur des hinteren Tibiakantenfragments wird Volk-

\begin{tabular}{lll} 
Tab. 1 & Klassifikation nach Danis-Weber. \\
$\begin{array}{l}\text { Frak- } \\
\text { turtyp }\end{array}$ & $\begin{array}{l}\text { Höhe der Fibula- } \\
\text { fraktur }\end{array}$ & $\begin{array}{l}\text { Syndesmo- } \\
\text { senstabilität }\end{array}$ \\
\hline A & infrasyndesmal & intakt \\
\hline B & transsyndesmal & fraglich intakt \\
\hline C & suprasyndesmal & nicht intakt
\end{tabular}




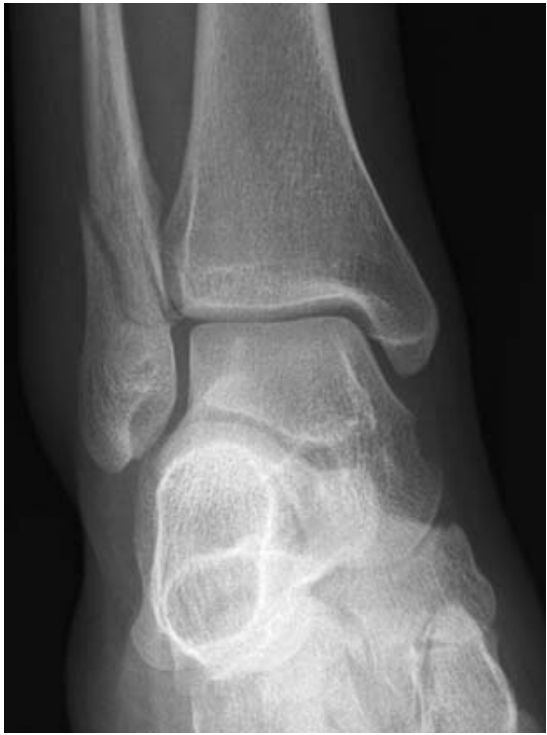

Abb. 1 A.-p.-Röntgenaufnahme einer Weber-B-Fraktur.

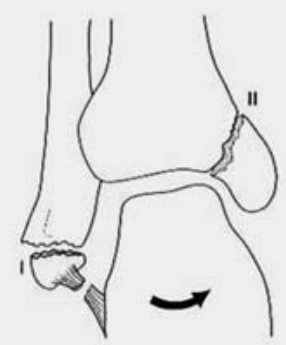

Supinationsfraktur

(Supination/Adduktion $=$ SA Type)

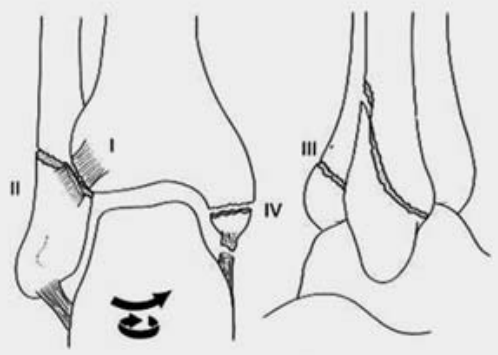

Supinations-/Außenrotationsfraktur (Supination/Eversions fraktur $=$ SE fraktur type)

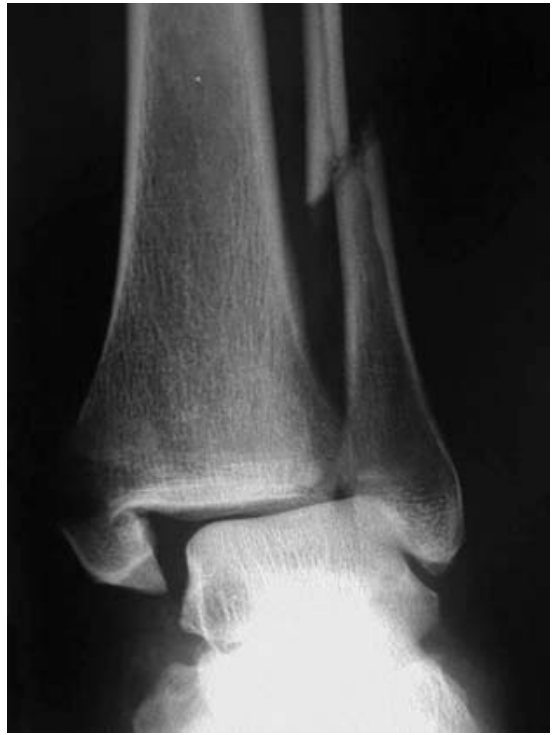

Abb. 2 A.-p.-Röntgenaufnahme einer Weber-C-Fraktur.

mann-Fraktur genannt, obwohl sich Herr Volkmann nachweislich nicht mit der Thematik auseinandergesetzt hat.

Genetische Klassifikation nach Lauge-Hansen (1942)

Hierbei handelt es sich um eine Fraktureinteilung, die den Unfallmechanismus zugrunde legt. Die Sprunggelenkverletzungen werden in 4 Grundtypen eingeteilt, je nach Stellung des Fußes zum Unfallzeitpunkt (Abb. 3 und Tab. 2).

Es werden röntgenologisch klar differenzierbare Typen und Schweregrade beschrieben, die Auskunft über begleitende Bandverletzungen geben. Diese Klassifikation ist im skandinavischen und angloamerikanischen Sprachraum weit verbreitet. Sie ermöglicht eine Analyse des Verletzungsmusters, indem sie ein Verständnis für die Ursache und das Ausmaß der osteoligamentären Verletzung schafft. Sie ist bedeutsam für die Technik der geschlossenen Reposition und die Planung des operativen Vorgehens. Ihre Kenntnis ist somit hilfreich für die Operationsstrategie; somit sollte sich jeder mit Sprunggelenkfrakturen befasste Arzt damit beschäftigen.

\section{Supinations-Adduktions-Fraktur (SA-Fraktur) (Abb. 4)}

Verletzung unterhalb der Syndesmose und damit nicht Gegenstand dieser Arbeit.

Pronations-Abduktions-Fraktur (PA-Fraktur) (Abb. 5)

Stadium 1

- Innenbandruptur oder waagerechte Fraktur des Innenknöchels

Stadium 2

- knöcherner/ligamentärer Ausriss der vorderen/hinteren Syndesmose

- teilweise mit relevanter Syndesmoseninstabilität

Stadium 3

- indirekte, irreguläre Fibulafraktur auf Höhe der Syndesmose bei forcierter Valgusstellung des Talus (lateraler Biegungskeil, zentrale Trümmerzone)

\section{Supinations-Eversions-Fraktur (SE-Fraktur) (Abb. 6)}

Die Verletzung beginnt an der vorderen Syndesmose und läuft über die Fibula, das posteriore Kantendreieck zum Innenknöchel. 


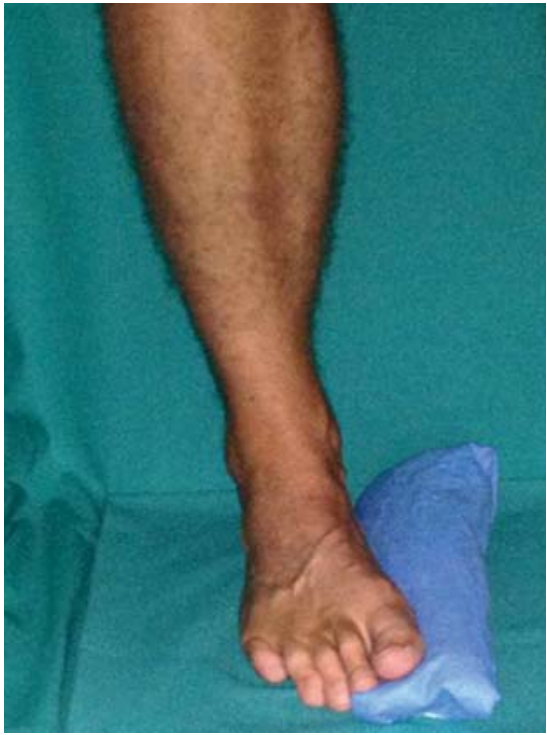

Abb. 4 Fußstellung bei Supinations-Adduktions-Verletzung.

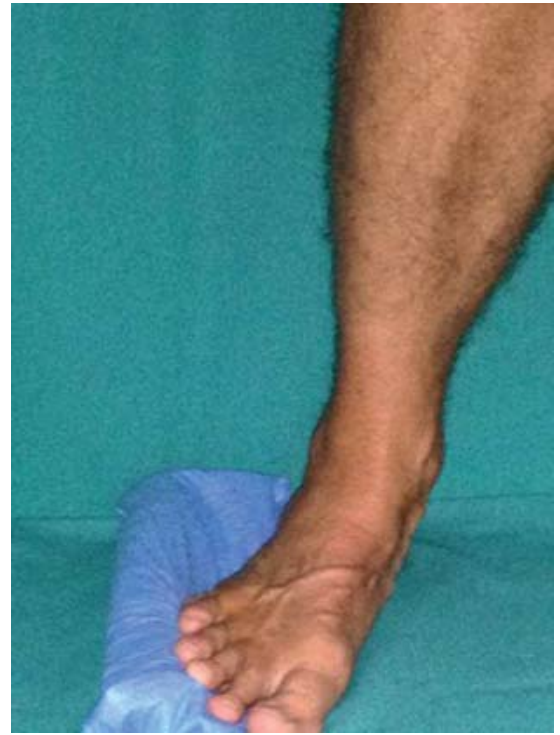

Abb. 5 Fußstellung bei Pronations-Abduktions-Verletzung.

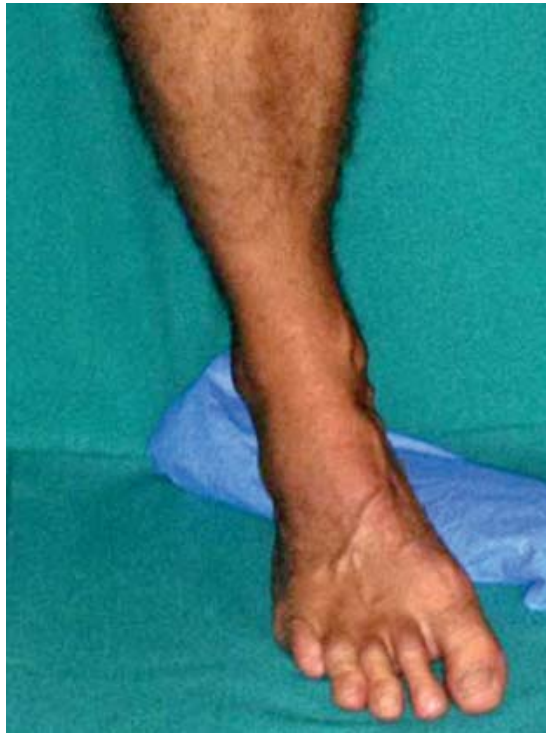

Abb. 6 Fußstellung bei Supinations-Eversions-Verletzung.

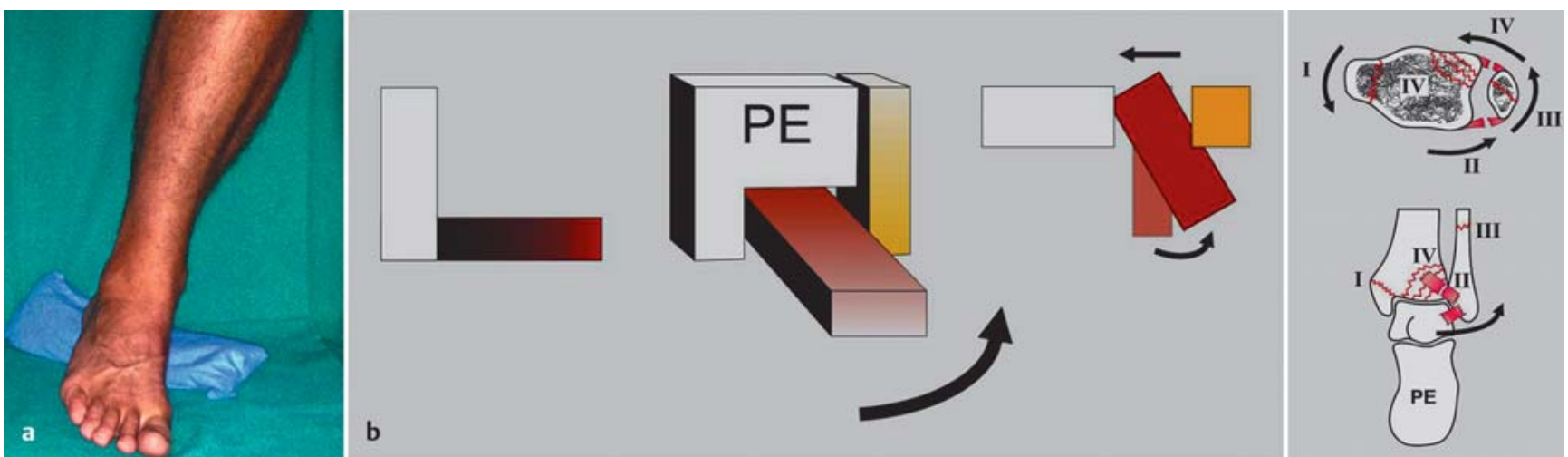

Abb. 7 a und b a Fußstellung bei Pronations-Eversions-Verletzung. b Schematische Darstellung des Kraftverlaufs einer Pronations-Eversions-Verletzung.

Stadium 1

- Ruptur oder knöcherner Ausriss der vorderen Syndesmose (tibial: Tillaux/ Chaput, fibular: Wagstaffe-Fragment)

Stadium 2

- Spiralfraktur der Fibula auf Syndesmosenhöhe

Stadium 3

- Ruptur der hinteren Syndesmose oder knöcherner Ausriss eines hinteren „Volkmann-Dreiecks“

- Instabilität der Malleolengabel

Stadium 4

- Fraktur des Innenknöchels bzw. Deltabandruptur

\section{Pronations-Eversions-Fraktur (PE-Fraktur) (Abb. 7)}

Die Verletzung nimmt ihren Ausgang am Innenknöchel und verläuft über die vordere Syndesmose, die Fibula zum posterolateralen Kantendreieck.
Stadium 1

- Ruptur des Lig. deltoideum oder Abrissfraktur des Innenknöchels

Stadium 2

- Ruptur der vorderen Syndesmose bzw. knöcherner Ausriss (tibial: Tillaux/ Chaput, fibular: Wagstaffe-Fragment)

- Ruptur der Membrana interossea cruris

Stadium 3

- Fraktur der Fibula oberhalb der Syndesmose

- Sonderform: Maisonneuve-Fraktur (hohe Fibulafraktur, ggf. Luxation des Fibulaköpfchens)

Stadium 4

- Ruptur der hinteren Syndesmose oder knöcherner Ausriss („Volkmann-Dreieck“)

Alice Wichelhaus und Thomas Mittlmeier: OSG-Frakturen Weber-B/C: was ist Standard?

\section{Diagnostik}

Frakturen des oberen Sprunggelenks werden durch eine klinische Untersuchung einschließlich Erhebung des Unfallmechanismus diagnostiziert. Die klinische Untersuchung hat einen hohen Stellenwert. Der Syndesmosenschmerz und der Schmerz bei Außenrotation haben eine so hohe Sensitivität zur Erkennung von Frakturen und Syndesmosenverletzungen, dass die notwendigen Röntgenuntersuchungen reduziert werden können. Erst nach radiologischem Frakturausschluss sollte eine Prüfung der Band- und Syndesmosenstabilität erfolgen. Während der klinischen Untersuchung wird die Schwellung beurteilt, ein Kompartmentsyndrom ausgeschlossen, Hämatomverfärbungen, Spannungsblasen, Schürfungen, Hautkontusionen, offene Wunden registriert und auf einen Druckschmerz der Fibula von der Spitze 


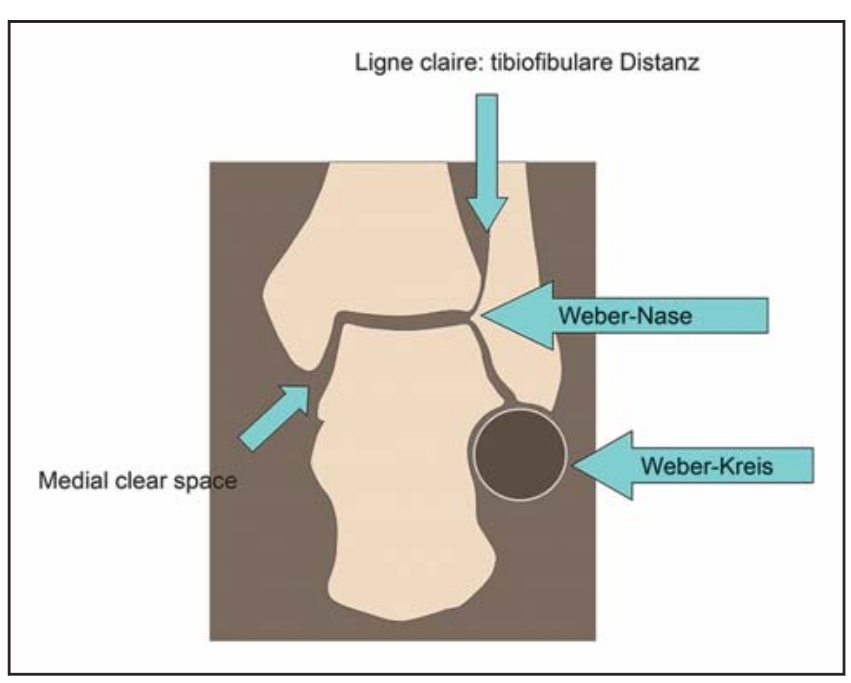

Abb. 8 Landmarks zur Beurteilung eines Röntgenbilds des OSG.

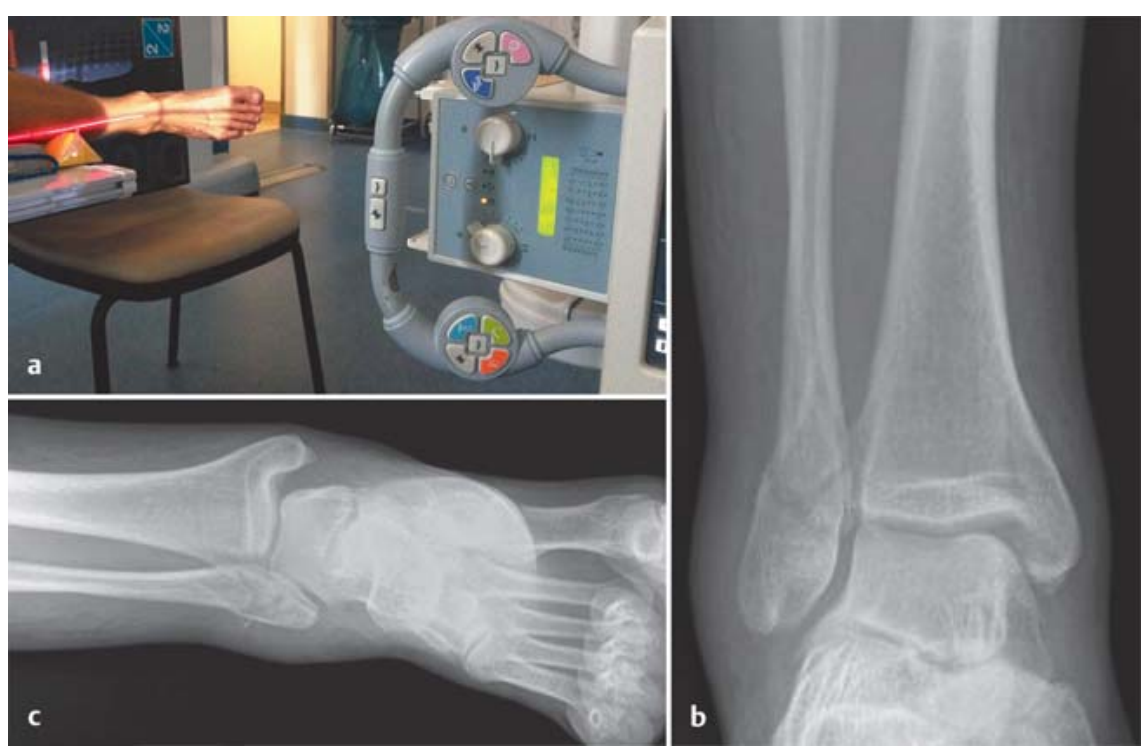

Abb.9a bis c a Patientenposition zum „gravity-stress“-Röntgen. b A.-p.-Röntgenaufnahme einer OSG-Fraktur. c „gravity-stress“-Röntgenaufnahme mit Beweis der Syndesmoseninsuffizienz.

bis zum Fibulaköpfchen geachtet. Der Weichteilschaden hat Einfluss auf das Behandlungsergebnis.

\section{Radiologische Diagnostik}

Standard sind Röntgenaufnahmen des oberen Sprunggelenks in 2 Ebenen, wobei die anteroposteriore Röntgenaufnahme in $20^{\circ}$ Innenrotation des Unterschenkels angefertigt wird. Die 2. Ebene ist eine exakt seitlich eingestellte Aufnahme des OSG. Zur Beurteilung sind die in Abb. 8 gezeigten Landmarks geeignet. Ist die Weite des fibulotibialen Abstands $1 \mathrm{~cm}$ oberhalb des Gelenkspalts (sog. „ligne claire“) über $5 \mathrm{~mm}$, so ist dies ein Hinweis für eine Syndesmoseninstabilität. Medial sollte der Abstand zwischen
Tibia und Talus nicht erweitert sein („medial clear space“).

Eine Verschiebung des Talus nach lateral um $>2 \mathrm{~mm}$ stellt ein Kriterium für eine manifeste Instabilität dar.

Die „Weber-Nase“ zeigt bei korrekter Fibulalänge auf das Tibia-Plafond und die Fortsetzung der Kontur der Fibulaspitze am Processus lateralis tali bildet den sog. Weber-Kreis.

Eine CT-Untersuchung ist gelegentlich notwendig und sinnvoll, um begleitende Talusfrakturen zu detektieren, die Größe und die Fehlstellung des posterolateralen Kantendreiecks (sog. VolkmannDreieck) zu bestimmen oder um die Ver- sorgung komplexer Frakturen besser planen zu können.

Eine MRT-Untersuchung kann Aufschluss über ligamentäre Verletzungen geben. Zur Frakturdiagnostik ist das MRT nur eingeschränkt geeignet und wird hauptsächlich zur Abgrenzung von Stressfrakturen und pathologischen Frakturen eingesetzt. In der Notfalldiagnostik hat eine MR-Tomografie keinen Stellenwert.

\section{Stressaufnahmen}

Zur sicheren Prüfung der Syndesmosenstabilität, insbesondere zur Differenzierung stabiler von instabilen SupinationsEversions-Frakturen und somit zur Therapiewahl, kann der sog. „gravitystress“-Test (Abb.9) eingesetzt werden. Der verletzte Fuß hängt dabei passiv über die Unterlage hinaus. Eine Instabilität der Knöchelgabel kann bei einer so angefertigten Röntgenaufnahme gut und für den Patienten schonend dargestellt werden.

\section{Erstbehandlung}

Eine instabile oder dislozierte Fraktur des oberen Sprunggelenks wird im Unterschenkelgipsverband retiniert. Bei offensichtlicher Fehlstellung soll so zügig wie möglich die Reposition unter Längszug erfolgen. Hierzu ist meist eine Analgosedierung notwendig. Je länger die Luxation besteht, umso schwerer ist das Ausmaß der zu erwartenden Weichteilund Knorpelschädigung. Nach Reposition wird ein Gipsverband (gespaltener Unterschenkelgips oder dorsale Unterschenkelgipsschiene) angelegt, wenn die Hautverhältnisse dies zulassen. Die operative Sofortversorgung der Fraktur innerhalb der ersten 8 Stunden nach Unfall wird nicht immer organisierbar sein, hat aber klare Vorteile gegenüber der verzögerten Versorgung. Sie ist wesentlich schonender für die das Sprunggelenk umgebenden Weichteile, das Frakturhämatom läuft $\mathrm{ab}$, die Infekt- und Hautnekrosegefahr sinken.

Bei nicht retinierbaren Frakturen und bei höhergradigem Weichteilschaden sollte ein gelenküberbrückender Fixateur externe (mit Kalkaneus-Pin) angelegt werden und die definitive osteosynthetische Versorgung erfolgt nach Weichteilkonsolidierung. 


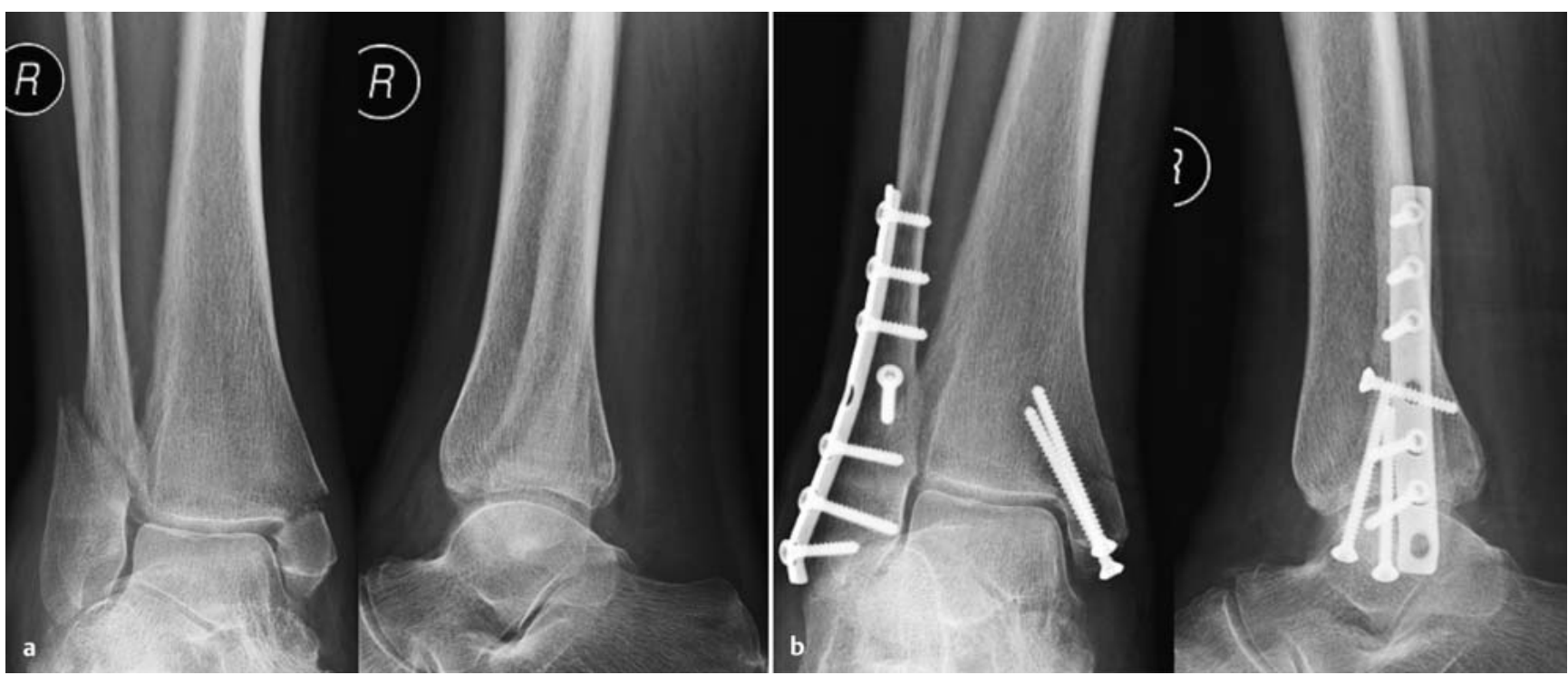

Abb.10a und b a Präoperative Aufnahmen einer trimalleolaren Weber-B-Fraktur. b Postoperative Aufnahmen einer trimalleolaren WeberB-Fraktur.

\section{Indikationen zur Operation}

Nur stabile, nicht dislozierte Frakturen (Fragmentverschiebung $<2 \mathrm{~mm}$ ) können konservativ behandelt werden. Schwerkraftgestützte Stressaufnahmen oder Belastungsaufnahmen im Stehen sind für die Entscheidungsfindung hilfreich, also etwa die Differenzierung einer SE-2Fraktur und einer SE-3- oder-4-Fraktur, wobei letztere sich nicht für die konservative Therapie eignen. Eine ligamentäre oder knöcherne Syndesmoseninstabilität und Verletzungen des medialen Kompartiments müssen somit sicher ausgeschlossen sein. In seltenen Fällen liegen allgemeine Kontraindikationen gegen eine Operation/Narkose vor, die ein konservatives Vorgehen notwendig machen.

Die operative Therapie ist für alle offenen Frakturen, für Frakturen mit Gefäßoder Nervenverletzung, für dislozierte, instabile und nicht retinierbare Frakturen indiziert, ebenso bei höhergradigem geschlossenen Weichteilschaden.

Definitionsgemäß ist jede Fraktur des oberen Sprunggelenks eine Fraktur mit Gelenkbeteiligung.

Die operative Therapie ist dabei erwiesenermaßen (Evidenzniveau 2b) der konservativen überlegen. Jede MaisonneuveVerletzung gilt als instabil, ebenso isolierte Syndesmosenverletzungen bei radiologisch nachgewiesener Malleolengabelinsuffizien-

\section{Risiken und Komplikationen}

Für konservative und operative Maßnahmen gibt es allgemeine sowie spezielle Risiken, über die der Patient sorgfältig aufgeklärt werden muss.

\section{Akute Komplikationen}

- Kompartmentsyndrom

- Schmerzen

- Druckstellen im Stützverband

- Thrombose und Embolie

- Peronäusläsion

- Entwicklung eines „complex regional pain syndrome“ (CRPS I/Morbus Sudeck)

- Durchblutungsstörungen

- sekundäre Dislokation

- Infektionen (in bis zu 2\% der Fälle)

- Wundheilungsstörungen, Wundrandnekrose und Ausbildung eines Wundhämatoms

- Implantatlockerung, -ausbruch, Schraubenfehllage

\section{Langfristige Komplikationen}

- Einsteifung des Gelenks

- Verbleib von Bewegungseinschränkungen

- Arthrosebildung (Früh- und Spätformen des Verschleißes), insbesondere bei bleibenden Fehlstellungen (in 10\% der Fälle)

- Schmerzen

- chronische Schwellung

- Atrophie von Knochen und Muskeln, ausbleibende Knochenbruchheilung mit Pseudarthrose (sehr selten)

\section{OP-Technik}

Ziel der operativen Behandlung ist die anatomische Reposition und Retention der Frakturfragmente bei Ermöglichung einer frühfunktionellen Nachbehandlung. Die Gelenkflächenkongruenz wird wiederhergestellt sowie Länge, Achse und Rotation, ebenso die ligamentäre Stabilität der Knöchelgabel. Meist erfolgt die Operation in Rückenlage, seltener in Bauch- oder Halbseitenlage bei notwendigem dorsalen Zugang, in Allgemeinoder Spinalanästhesie. Eine perioperative Single-Shot-Antibiose wird empfohlen. Am Oberschenkel wird eine Blutsperrenmanschette angelegt, um in Blutsperre oder -leere operieren zu können. Fuß und Unterschenkel werden beweglich abgedeckt.

Man benötigt folgendes Instrumentarium:

- Grundinstrumentarium für Knochenund Weichteilchirurgie

- Repositionszangen, gelegentlich Arthrodesenspreizer

- Konventionelle und winkelstabile Platten und Schrauben aus dem Kleinfragmentinstrumentarium

- Kirschner-Drähte, ggf. Zuggurtungsset

- bei zweizeitigem Vorgehen: Fixateur externe

- Bildwandler, wenn vorhanden 3-DBildverstärker (3-D-BV) 


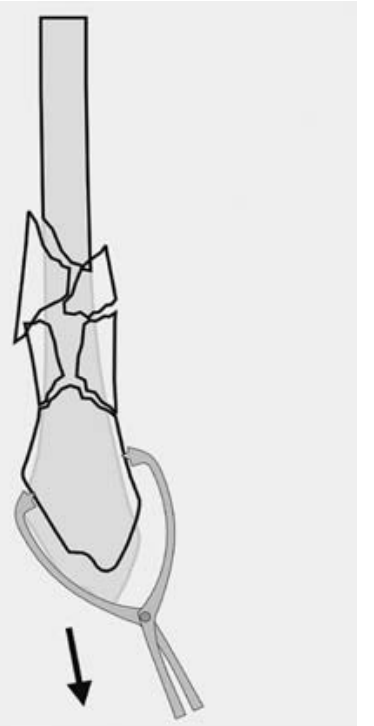

Abb.11 Schematische Darstellung des Repositionsmanövers bei Weber-C-Fraktur mit Repositionszange.

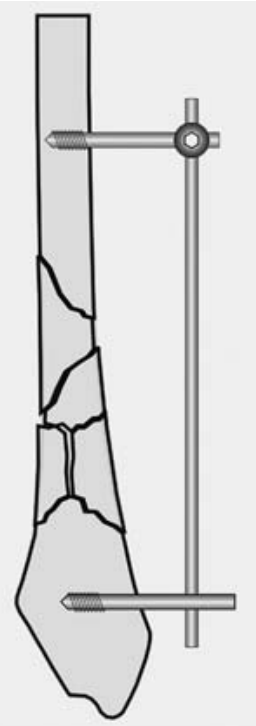

Abb.12 Schematische Darstellung des Repositionsmanövers bei Weber-C-Fraktur mit temporärem Fixateur externe.
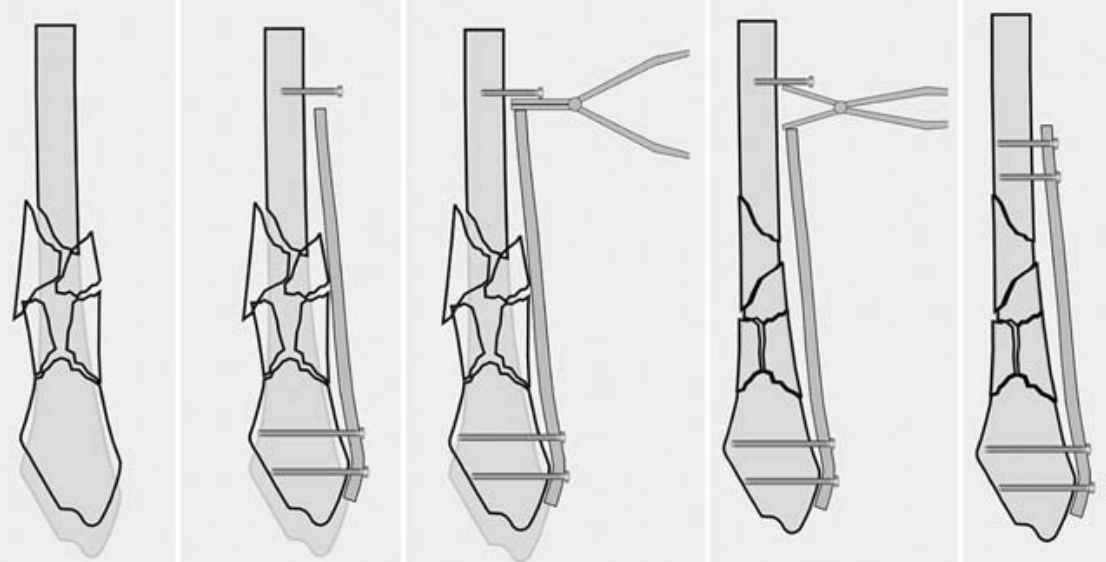

Abb. 13 Schematische Darstellung des Repositionsmanövers bei Weber-C-Fraktur mit Arthrodesenspreizer und „Push-and-pull“-Technik.

\section{Chirurgische Strategie}

\section{Osteosynthese der Fibulafraktur}

Distale Fibulafrakturen (Typ Weber B)

Die Außenknöchelfraktur wird über eine laterale Längsinzision versorgt. Auf eine genaue Längen- und Torsionswiederherstellung der Fibula ist zu achten. Kriterien für die korrekte Reposition sind der Weber-Kreis und die Weber-Nase. Die Reposition wird zunächst mit einer spitzen Repositionszange gehalten. Dann wird die Zugschraube senkrecht zur Fraktur gebohrt, um eine Kompression zwischen den Fragmenten zu erzeugen. In der Regel wird zur weiteren Sicherung zusätzlich eine Drittelrohrplatte angebracht (Abb. 10).

Die Platte ist lateral oder dorsolateral an der Fibula anzubringen.

Die dorsale Plattenlage bringt eine bessere Weichteildeckung mit sich. Bei osteoporotischem Knochen sollten winkelstabile Platten bevorzugt verwendet werden. Die Platte wird der Fibula anmodelliert und dann sukzessive mit Kortikalisschrauben besetzt, wobei darauf zu achten ist, dass die Schrauben distal die mediale Kortikalis nicht überragen und damit zu Knorpelschäden am Talus führen. Distal können auch Spongiosaschrauben eingebracht werden.

\section{Hohe Fibulafraktur}

Bei langstreckigen Spiralfrakturen ist es sinnvoll, 2 oder mehr Zugschrauben zu verwenden. Bei Trümmerzonen, wie sie bei osteoporotischem Knochen häufig anzutreffen sind, wird eher eine Rekonstruktionsplatte zur Anwendung kommen. Die Platte wird nicht vorgebogen und überbrückt den mehrfragmentären Bereich als sog. Brückenplatte. Zur Reposition der Fraktur kann direkter Zug angewendet werden durch eine an der Außenknöchelspitze angebrachte Repositionszange (Abb.11). Hierdurch läuft man Gefahr, mit der Repositionszange das distale Fibulaende zusätzlich zu frakturieren. Alternativ kann eine indirekte Reposition über einen temporär angebrachten Fixateur externe erfolgen (Abb. 12). Eine weitere Repositionsmethode ist die sog. „Push-and-pull“Technik, die in Abb. 13 gezeigt wird. Es wird zunächst die Brückenplatte angebracht und distal fixiert. Dann wird proximal des proximalen Plattenendes eine Schraube in die Fibula eingebracht, um zwischen diese Schraube und das Plattenende einen Arthrodesenspreizer setzen zu können, über den dann die Frakturreposition erleichtert wird.

\section{Versorgung der Syndesmosenverletzung}

Bei jeder Weber-B- oder -C-Fraktur ist intraoperativ die Stabilität der Syndesmose zu überprüfen.

Hierzu wird mit einem Einzinker Zug nach laterodorsal auf die Fibula ausgeübt und unter Durchleuchtung die Erweiterung des tibiofibularen und talotibialen Gelenkspalts beurteilt. Bei nachgewiesener Syndesmoseninstabilität (entsprechend den Frakturtypen nach LaugeHansen PA 3 und 4, SE 3 und 4, PE 1 und 2) wird die Fibula in der Inzisur der Tibia eingestellt. Hierzu wird eine große Repositionszange mit Spitzen auf Höhe der Gelenklinie vom Innenknöchel auf den Außenknöchel („tip-to-toe“) aufgesetzt. Dann wird eine Stellschraube gebohrt $2 \mathrm{~cm}$ oberhalb des Tibia-Plafonds parallel zur Gelenklinie etwa $30^{\circ}$ von dorsal nach ventral ansteigend, sodass typischerweise 3 Kortikales gefasst werden. Dieser Winkel entspricht der Rotationsebene des Sprunggelenks. Die Schraube kann eine der plattenbesetzenden Schrauben ersetzen, soll aber keinesfalls durch das distale Fibulotibialgelenk verlaufen. Die Stellschraube ist keine Zugschraube. Druck auf das Gelenk soll durch die Einbringung der Stellschraube nicht aus- 


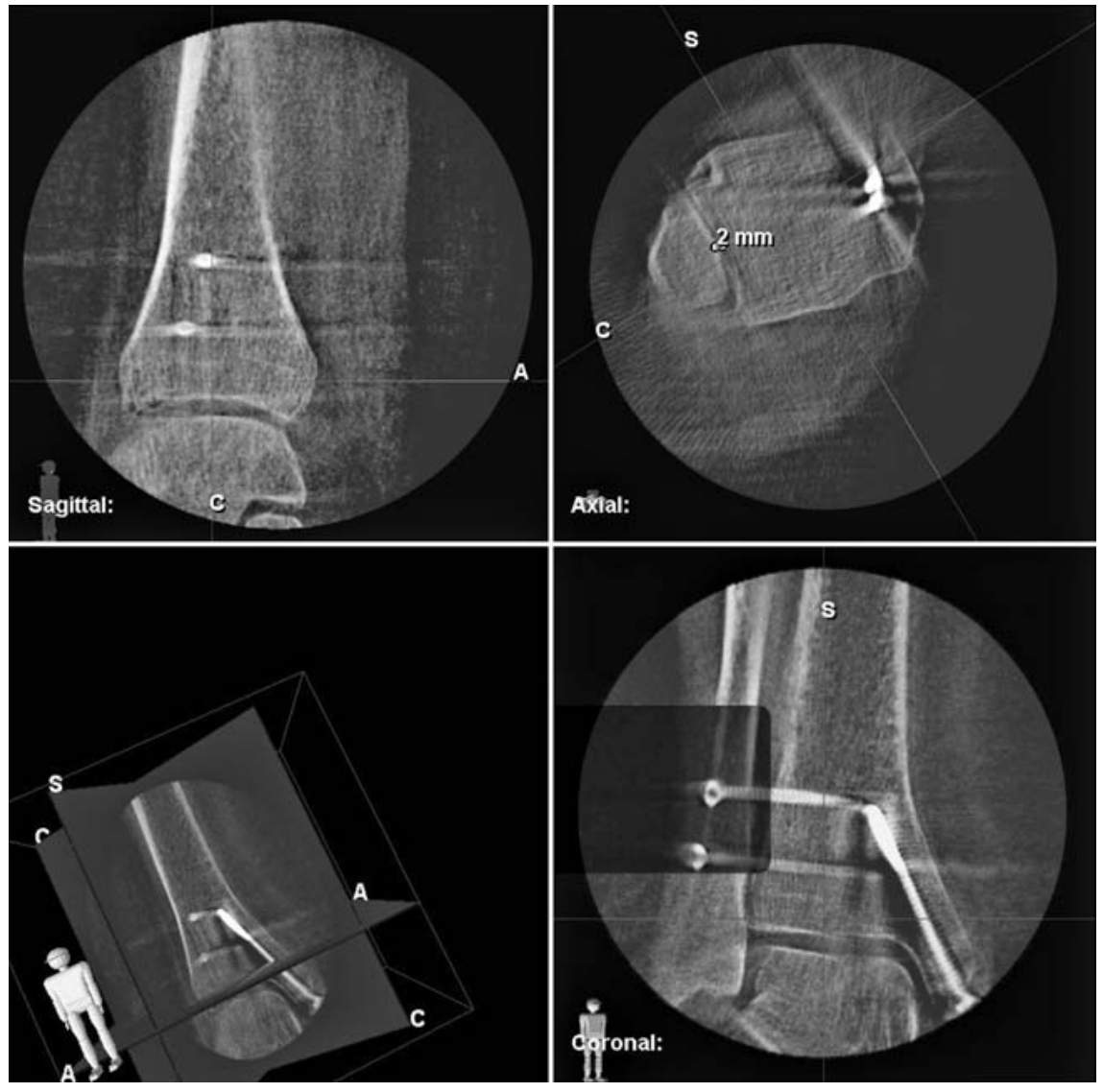

Abb. 14 Intraoperative Repositionskontrolle und Einstellung der Fibula in der Inzisur mit dem 3-D-BV.

geübt werden, sie ist auch kein Repositionsinstrument. Alternative Techniken mittels flexibler Implantate oder resorbierbarer Schrauben können grundsätzlich denselben Zweck wie die Stellschraube erfüllen, sind aber kostspieliger.

Eine intraoperative 3-D-Bildwandler-Untersuchung ist zur korrekten Lagebestimmung und Repositionsbeurteilung sehr hilfreich (Abb. 14).
In Studien konnte gezeigt werden, dass intraoperativ in etwa $20 \%$ der Fälle Fehlpositionierungen der Fibula in der Tibiainzisur, zusätzliche Fragmente und Implantatfehllagen festgestellt werden. Im eigenen Krankengut konnte die Revisionsrate durch intraoperative Anwendung des 3-D-BVs auf nahezu 0\% gesenkt werden.

Knöchern kann die Syndesmose mit einem Fragment an der Fibula ausreißen.
Dieses Fragment wird Wagstaffe-Fragment genannt. Die Refixation erfolgt i.d.R. mit einer Schraubenosteosynthese. Der knöcherne Syndesmosenausriss an der Tibia wird mit Tubercule de Tillaux-Chaput bezeichnet. Auch dieses Fragment wird schraubenosteosynthetisch versorgt. Bei sehr kleinen oder schaligen Fragmenten kann eine transossäre Naht oder Nahtankerrefixation erforderlich sein (Abb.15). Werden solche Fragmente nicht erkannt oder nicht refixiert, kann das die korrekte Einstellung der Fibula in der Inzisur verhindern.

Ob bei einer Maisonneuve-Fraktur eine Osteosynthese der kniegelenksnahen Fibulafraktur erfolgen muss oder nicht, ist strittig. Bei wenig dislozierten Frakturen kann darauf verzichtet werden. Zur Stabilisierung der Syndesmose werden dann 2 Stellschrauben eingebracht. Bei komplexeren Fibulafrakturen wird die korrekte Längen- und Rotationseinstellung der Fibula erleichtert durch eine direkte Reposition der proximalen Fibulafraktur (Abb. 16a, b). Der N. peronaeus muss in seinem frakturnahen Verlauf geschont werden. Liegt eine primäre Peronäsläsion vor, sollte der Nerv ohnehin dargestellt, neurolysiert oder bei Zerreißung mit Interponaten versorgt werden.

Osteosynthese der Innenknöchelfraktur

Verschobene Innenknöchelfrakturen, die meist im Bereich des Gelenkwinkels lokalisiert sind, werden geschlossen oder offen reponiert und mit Zugschrauben versorgt, bei schlechter Knochensubstanz können Unterlegscheiben verwendet werden. Alternativverfahren ist die Zuggurtungsosteosynthese.

Unversorgt führen Innenknöchelfrakturen zu einer Varusfehlstellung des Talus.
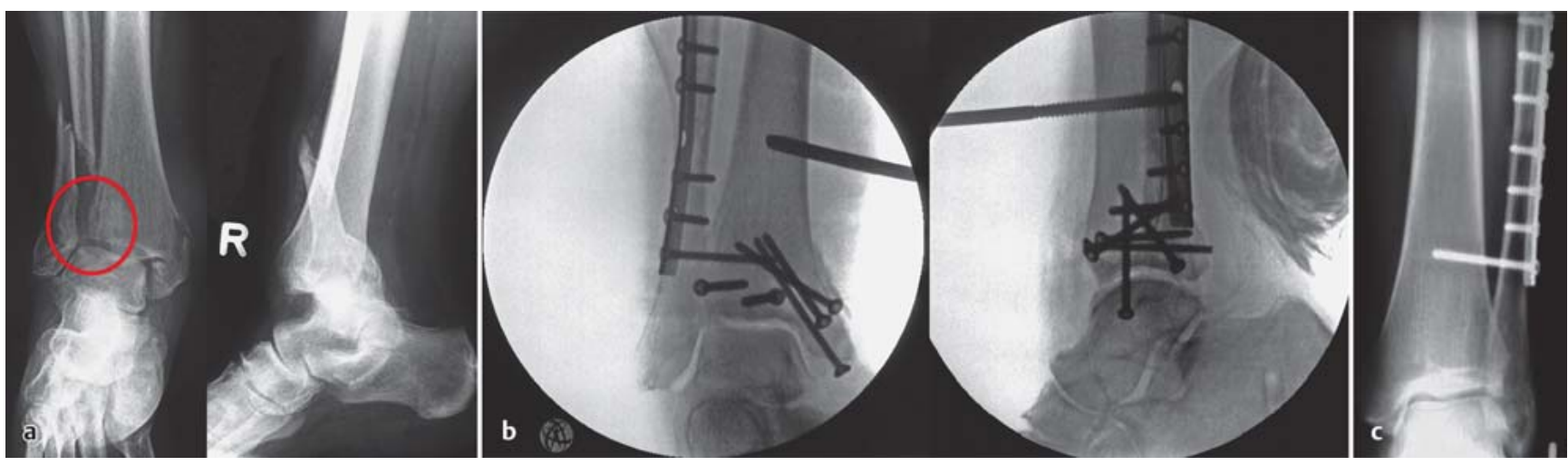

Abb. 15a bis c a Tubercule de Chaput-Fragment bei Pronations-Eversions-Fraktur präoperativ. b Tubercule de Chaput-Fragment postoperativ. c Postoperatives Röntgenbild a.-p. mit korrekt eingestellter Sprunggelenkgabel. 


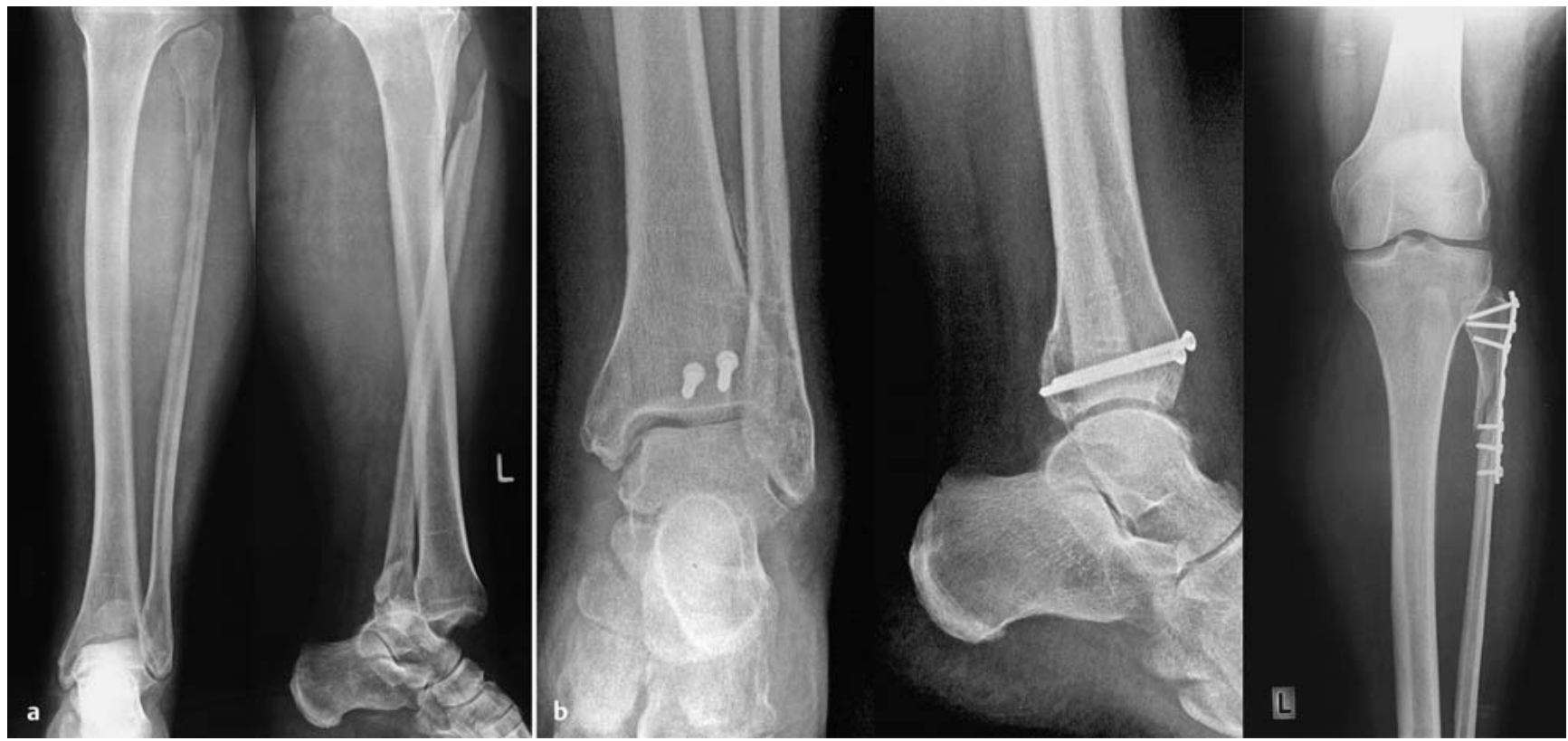

Abb. 16a und b a Röntgenaufnahmen einer hohen Fibulafraktur präoperativ. b Röntgenaufnahmen einer hohen Fibulafraktur postoperativ.
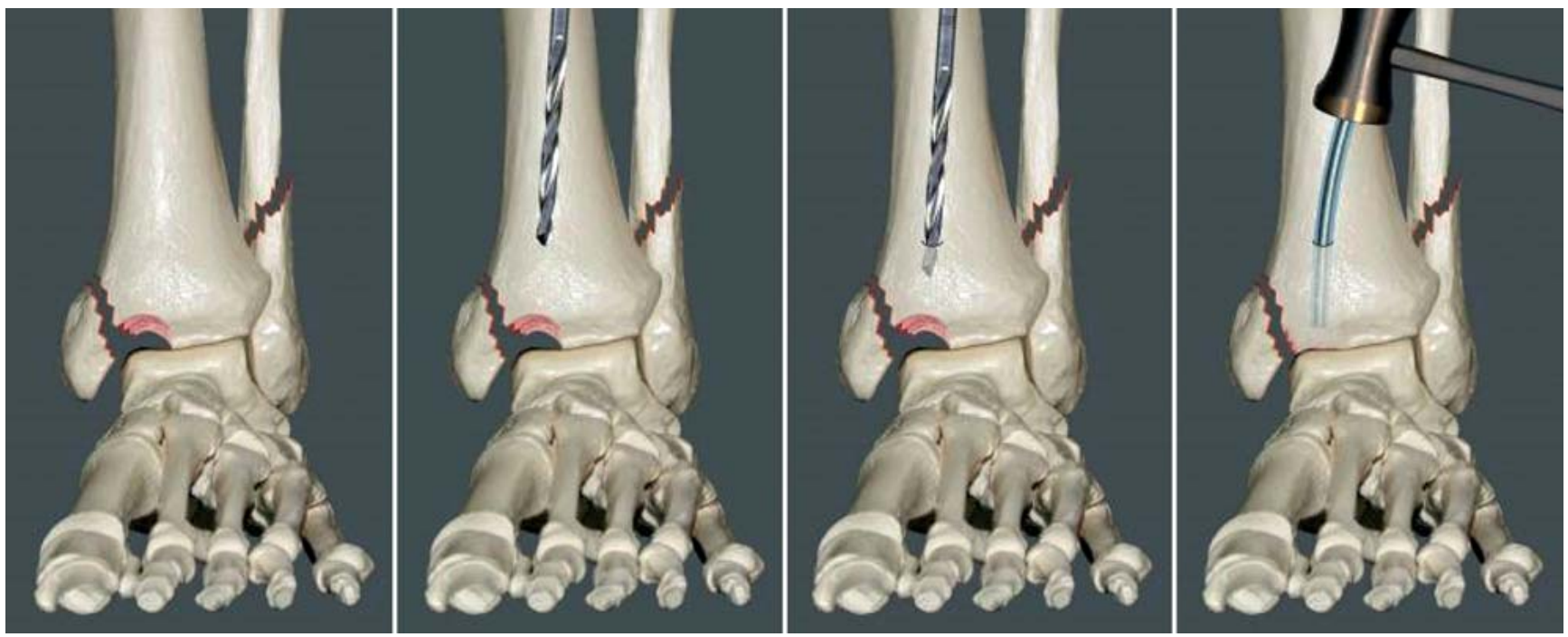

Abb. 17 Schematische Darstellung der Reposition einer anteromedialen Impression.

Der Zugang wird epimalleolar gerade oder leicht geschwungen angelegt.

Bei einer Supinations-Adduktions-Fraktur findet sich häufig eine senkrecht verlaufende Innenknöchelfraktur, die mit 1-2 waagrecht von medial eingebrachten Zugschrauben osteosynthetisch versorgt wird. Anteromediale Impressionen des Tibia-Plafonds sollten angehoben und ggf. mit Spongiosa unterfüttert werden. Ein Trick zur Reposition ist in Abb. 17 dargestellt. Gelegentlich wird auch an dieser Lokalisation eine Neutralisationsplatte zur sicheren Retention benötigt.
Eine reine Ruptur des Lig. deltoideum bedarf keiner zusätzlichen Naht. Bei korrekt eingestellter Knöchelgabel bilden die Bandenden eine ausreichend stabile Narbe auch ohne Bandnaht.

\section{Osteosynthese der Tibiahinterkante}

In den meisten Lehrbüchern wird die Meinung vertreten, dass eine Osteosynthese der hinteren Tibiakante notwendig ist, wenn die Fragmentgröße mehr als ein Viertel bis ein Drittel der Gelenkfläche beträgt. Bei Gelenkstufen von $\geq 2 \mathrm{~mm}$ sollten aber unserer Ansicht nach auch kleinere Fragmente reponiert werden, da das sog. hintere Kantendreieck Indikator ist für eine Syndesmosen- instabilität und unversorgt eine Subluxation des Talus ermöglicht.

Günstig ist es in vielen Fällen, die Reposition des hinteren Tibiakantenfragments vor der Osteosynthese von Innen- und Außenknöchel vorzunehmen, da dann eine direkte Kontrolle der Reposition durch die Fibulafraktur möglich ist. Auch radiologisch ist die Repositionskontrolle im seitlichen Strahlengang einfacher, da keine Überlagerungen durch Osteosynthesematerial die Bildbeurteilung stören. Allerdings stellt sich bei instabilen Verletzungen das hintere Tibiakantenfragment nach korrekter Reposition der Fibula und des Innenknöchels wieder 


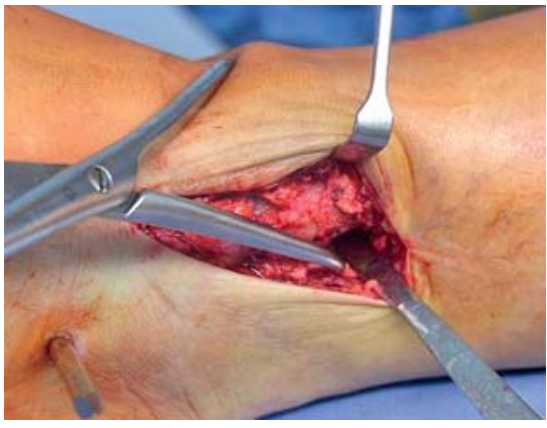

Abb. 18 Reposition des dorsalen Tibiakantenfragments, klinisches intraoperatives Bild.

gut ein (Repositionsindikator), sodass dann die Versorgung in anderer Reihenfolge günstiger sein kann.

Die indirekte Verschraubung des sog. Volkmann-Dreiecks ist bei ausreichend großem Fragment gut möglich.

Nach Frakturdarstellung und Säuberung über die laterale Inzision, die auch zur Fibulaosteosynthese genutzt wird, wird die Reposition mittels Raspatorium oder Hakenzug durchgeführt oder mit einer großen Repositionszange, die an den Peronäalsehnen vorbei hinter der Fibula auf das dorsale Fragment aufgesetzt wird (Abb. 18). Dann werden 2 Zugschrauben von ventral nach dorsal unter Bildwandlerkontrolle über einen kleinen ventralen Zugang senkrecht zur Frakturlinie eingebracht. Bei der ventralen Inzision sind die Strecksehnen, Äste des N. peronaeus und das ventrale Gefäß-NervenBündel zu schonen. Das Gewinde der Schraube soll komplett im dorsalen Fragment liegen. Hierzu muss es manchmal gekürzt werden. Ein zentrales Imprimat muss ausgeschlossen sein. Bei minderer Knochenqualität kann es sinnvoll sein, Unterlegscheiben zu verwenden.

Fragmente mit komplexerem Frakturmuster, Gelenkimpressionen oder interponierte Fragmente werden besser von dorsal unter direkter Sicht versorgt.

Hierzu liegt der Patient in Bauch- oder Seitenlage und es wird ein dorsolateraler Zugang angelegt. Der Hautschnitt liegt zwischen Achillessehne und den Peronäalsehnen und ist 5-8 cm lang. Die Unterschenkelfaszie wird durchtrennt, der N. suralis geschont und die Flexor-hallucis-longus-Sehne nach medial beiseite gehalten. Hierdurch wird das posteriore Gefäß-Nerven-Bündel geschützt. Die hintere Gelenkkapsel wird durchtrennt, sodass man das obere Sprunggelenk einsehen kann. Das hintere Kantenfragment
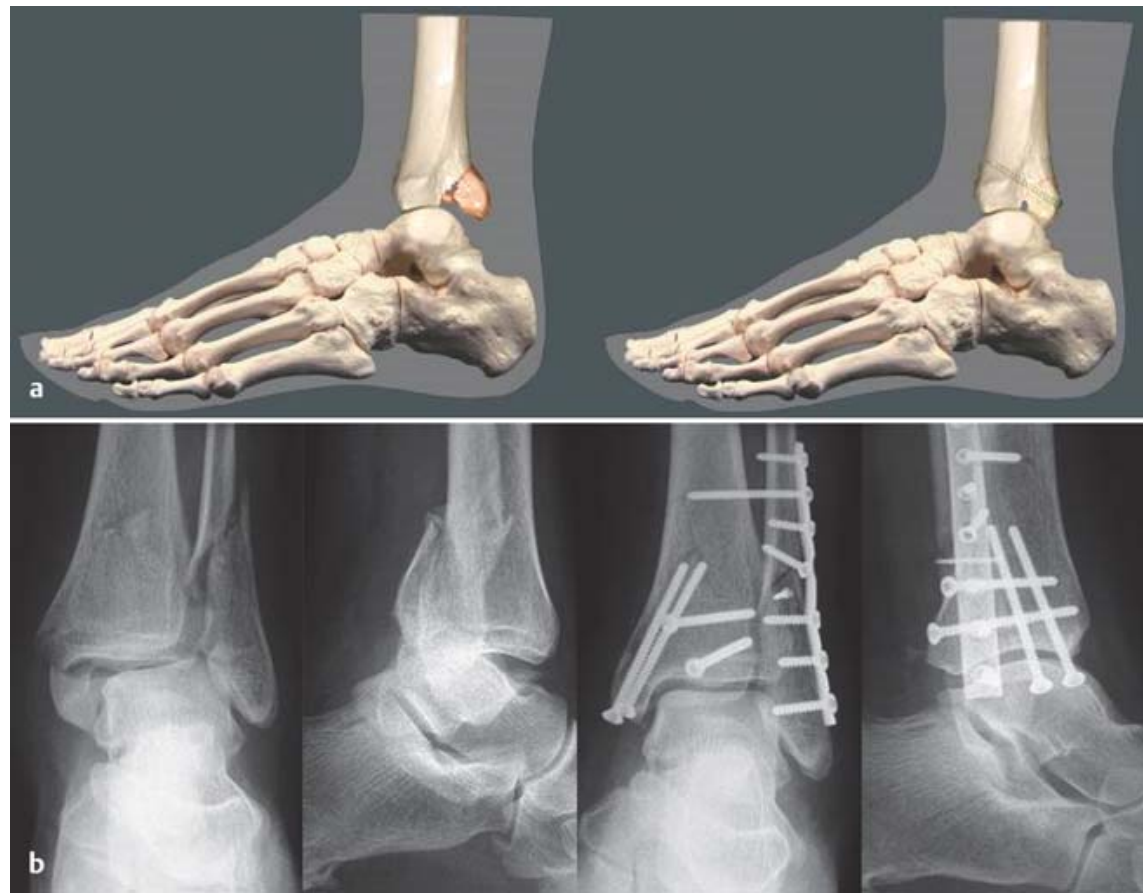

Abb. 19a und b a Schematische Darstellung der direkten Reposition des dorsalen Tibiakantenfragments. b Prä- und postoperative Röntgenaufnahmen einer Pronations-Eversions-Fraktur Stadium IV.

kann mit dem anhängenden posterioren Syndesmosenband weggeklappt werden. Ein Gelenkflächenimprimat wird angehoben, ggf. mit Spongiosa unterfüttert, die man aus der Tibiametaphyse gewinnen kann. Wenn nötig, wird die Reposition mit einem resorbierbaren Pin gesichert. Dann wird das hintere Kantenfragment reponiert. Die Osteosyntheseform ist abhängig von der Fragmentgröße. Es werden Zugschrauben mit oder ohne Unterlegscheiben oder Antigleitplatten aus dem Kleinfragmentinstrumentarium benutzt (Abb. 19a, b).

\section{Nachbehandlung}

Der 1. Verbandswechsel wird einschließlich Entfernung der eingebrachten Drainage am 2. postoperativen Tag durchgeführt. Selbstständiges Anpressen der Fußsohle gegen den stabilisierenden Verband unterstützt die Entleerung der venösen Plexus, mindert damit die Thrombosegefahr und hilft beim Abschwellen. In der Regel wird für 6 Wochen ruhig gestellt im Gipsverband oder einem orthetischen Gipsersatz, der den Vorteil der besseren Körperpflegemöglichkeit hat. Frühzeitig kann mit aktiven und passiven Bewegungsübungen des Sprunggelenks für die Dorsalextension und Plantarflexion begonnen werden, unabhängig davon, ob eine Stellschraube eingebracht wurde. Hierzu wird das Bein aus der Gipsschiene oder Orthese herausgenommen.

Die notwendige Teilbelastung von 15$20 \mathrm{~kg}$ Körpergewicht macht eine medikamentöse Thromboseprophylaxe erforderlich.

Eingebrachte Stellschrauben werden nach 6-8 Wochen entfernt.

Für diesen Eingriff ist meist eine Lokalanästhesie ausreichend. Erst nach Stellschraubenentfernung kann schrittweise zur Vollbelastung übergegangen werden. Sportfähigkeit wird etwa nach 3 Monaten erreicht.

Eine komplette Implantatentfernung ist nicht zwingend erforderlich, kann aber bei Konflikt mit dem Schaft oder Rand des Schuhes durch das mit wenig Weichteil bedeckte Osteosynthesematerial nach etwa 1 Jahr erfolgen.

\section{Schlussfolgerung}

Voraussetzung für die erfolgreiche Versorgung von Sprunggelenkfrakturen, ist die präoperative Analyse des Verletzungsausmaßes.

Das Verständnis und die Kenntnis der Lauge-Hansen-Klassifikation hilft hierbei jedem operativ Tätigen wesentlich 
weiter als die Limitierung auf die DanisWeber-Klassifikation. Die Wiederherstellung der Integrität des Knöchelkomplexes ist abhängig von der adäquaten Diagnose der Frakturelemente. Intraoperativ muss auf die korrekte Wiederherstellung der Fibulalänge und die Torsionseinstellung in der Tibiainzisur geachtet werden. Eine intraoperative 3-D-BV-Untersuchung oder ein postoperatives CT ist bei komplexeren Frakturen zur Erfolgskontrolle notwendig. Die Anlage eines Fixateur externe als Erstmaßnahme ermöglicht eine geplante definitive Versorgung, etwa bei kritischer Weichteilsituation. Wesentliches langfristiges Risiko ist die Entwicklung einer posttraumatischen Arthrose des oberen Sprunggelenks. Der Operateur kann dies beeinflussen, indem die Reposition so genau wie möglich vorgenommen wird. Gelenkstufen von mehr als $2 \mathrm{~mm}$ sollten nicht akzeptiert werden.

Ein höheres Stadium in der Lauge-Hansen-Klassifikation ist mit schlechteren Ergebnissen assoziiert.
Das Vorliegen einer Luxationsfraktur steigert das Risiko der Arthroseentwicklung. Chronische Syndesmoseninstabilitäten sind mit schlechteren funktionellen Ergebnissen gekoppelt.

\section{Literatur}

${ }^{1}$ AWMF-Leitlinien Sprunggelenkfraktur. Diagnostik und Therapie; Leitlinie der Deutschen Gesellschaften für Unfallchirurgie. AWMFRegister Nr.012/003. Im Internet: http:// www.awmf.org/leitlinien/aktuelle-leitlinien. html; Stand: 10.06.2014

2 Goost H, Wimmer MD, Burg A. Fractures of the ankle joint: investigation and treatment options. Dtsch Arztebl Int 2014; 111: 377-388

${ }^{3}$ Lauge-Hansen N. Fractures of the ankle II: combined experimental/surgical and experimental roentgenologic investigation. Arch Surg 1950; 60: 957-985

${ }^{4}$ Makwana NK, Bhowal B, Harper WM et al. Conservative versus operative treatment for displaced ankle fractures in patients over 55 years of age. A prospective, randomised study. J Bone Joint Surg Br 2001; 83: 525-529

${ }^{5}$ Miller SC. Late reconstruction after failed treatment for ankle fractures. Orthop Clin North Am 1995; 26: 363-373

${ }^{6}$ Rammelt S, Grass R, Zwipp H. Sprunggelenksfrakturen. Unfallchirurg 2008; 111: 421-438
${ }^{7}$ Rammelt S, Zwipp H, Grass R. Sprunggelenksfrakturen: operative Technik. Unfallchirurg 2008; 111: 439-447

${ }^{8}$ Rammelt S, Zwipp H, Mittlmeier T. Therapie der Sprunggelenksluxationsfrakturen vom Pronationstyp. Oper Orthop Traumatol 2013; 25: $273-291$

\section{Dr. Alice Wichelhaus}

Geschäftsführende Oberärztin der Abteilung für Unfall-, Hand- und Wiederherstellungschirurgie

alice.wichelhaus@med.uni-rostock.de

Univ.-Prof. Dr. Thomas Mittlmeier Leiter der Abteilung für Unfall-, Handund Wiederherstellungschirurgie

Chirurgische Klinik und Poliklinik Universitätsmedizin Rostock

Schillingallee 35

18055 Rostock

thomas.mittlmeier@ med.uni-rostock.de 